\title{
REFLEXIONES EN TORNO A LA FORMACIÓN DE UN ORGANISMO ASOCIATIVO QUE ACOJA Y REPRESENTE AL COLECTIVO DE TRABAJADORAS CULTURALES
}

\author{
Itziar Zorita Agirre \\ Universidad del País Vasco / Euskal Herriko Unibertsitatea, Dpto Comunicación Audiovisual y \\ Publicidad
}

\section{Resumen}

Existen múltiples asociaciones conformadas por artistas y trabajadoras culturales cuya misión es principalmente aunar esfuerzos para defender y reivindicar mejoras desde el punto de vista laboral, político, social y existencial. Sin embargo, siguen siendo pocas las trabajadoras culturales que se afilian a algún tipo de organismo estructurado y formal. Frente a esta realidad, ejemplos como la comunidad de InsultARTe, ofrecen alternativas complementarias para el encuentro entre profesionales de la cultura. Se trata de otro tipo de colectividad de carácter más informal que se establece en espacios digitales, desde los márgenes y en condiciones de una mayor precariedad en cuestión de recursos, visibilidad y capacidad de instaurarse como sujeto interlocutor social. El caso de InsultARTe plantea el debate sobre qué tipo de organización se podría crear, cuáles son los perfiles que conforman esa posible asociación o sobre cuál debería ser su funcionamiento y modelo organizacional.

\section{Palabras clave: TRABAJADORA CULTURAL; COMUNIDAD ABIERTA; INSULTARTE (ASOCIACIÓN CULTURAL)}

\section{REFLECTIONS ABOUT THE CREATION OF AN ASSOCIATION THAT WELCOMES AND REPRESENTS THE COLLECTIVE OF CULTURAL WORKERS}

\begin{abstract}
There are multiple associations built by artists and cultural workers whose mission is mainly to join forces to defend and demand improvements from a labor, political, social and existential point of view. However, there are still few cultural workers who join some kind of structured and formal associations. Faced with this reality, examples such as the InsultARTe community offer complementary alternatives for the meeting between cultural professionals. It is another type of community, more informal, that is established in digital spaces, from the margins and in conditions of bigger precariousness in terms of resources, visibility and ability to establish itself as a social interlocutor. The case of InsultARTe raises the discussion about what type of organization could be created, what are the professional profiles that could be included in this kind of community, its proper functioning or which organizational model should have.
\end{abstract} Keywords: CULTURAL WORKER; OPEN COMMUNITY; INSULTARTE (CUL-
TURAL ASSOCIATION)

Zorita Agirre, Itziar. 2019. "Reflexiones en torno a la formación de un organismo asociativo que acoja y represente al colectivo de trabajadoras culturales“. AusArt 7 (2): 49-59. D0I: 10.1387/ausart.21143

\section{AUSART}




\section{INTRODUCCIÓN}

Resulta complejo cuantificar y realizar un diagnóstico riguroso en torno a la situación profesional de las trabajadoras de la cultura, tanto si aludimos a las artistas, las creadoras, como a las otras funciones que se enmarcan dentro del trabajo de producción, comunicación, gestión o mediación que se desarrollan dentro del contexto cultural y, en concreto, la práctica artística. Tal y como se menciona en el exhaustivo estudio realizado por Marta Pérez Ibáñez e Isidro López-Aparicio (2018) en torno a la situación laboral de las trabajadoras de la cultura, la falta de un censo profesional supone un importante obstáculo a la hora de realizar una fotografía coherente y consecuente sobre la realidad de estas trabajadoras. La ausencia de datos fehacientes que cuantifiquen el número de profesionales del sector artístico-cultural, no hace más que añadir incertezas, diagnósticos parciales, además de determinar una mirada más especulativa a la hora de afrontar un estudio o análisis del contexto.

La categoría de trabajadora cultural es amplia, difícil de precisar y demanda de un escenario que establezca los márgenes de lo que incluimos y excluimos en la noción de trabajadora cultural. Pérez lbáñez y López-Aparicio detallan y exponen cuáles son algunos de los condicionantes atribuidos a la profesional cultural. En ese listado de funciones y consideraciones surge la primera encrucijada: ¿Dentro de qué régimen profesional o regímenes profesionales se sitúa este tipo de trabajadora? ¿Qué es aquello que consideramos como profesional entre todas las posibilidades de prácticas y haceres que existen dentro del trabajo cultural? La indefinición y la variedad de posibilidades que se abren en torno al hacer artístico/cultural obliga a incluir un sin fin de actividades y, por tanto, diferentes modelos de trabajadoras. En otras palabras, es posible encontrar trabajadoras culturales en más de un epígrafe de las que ofrecen tanto el sistema de Seguridad Social o las diferentes agencias tributarias, bien sea por cuenta ajena y/o propia. Por si esto no fuera suficiente, a esta heterogeneidad de registros profesionales, resulta oportuno incluir también dentro del trabajo cultural todas aquellas actividades que no operan desde una lógica de retribución económica. La complejidad de crear un censo que reúna y contabilice el número de trabajadoras culturales queda demostrada. La dificultad es obvia.

Sin embargo, existen junto con el estudio mencionado anteriormente otros tantos intentos y buenos ejemplos que arrojan algunas pistas sobre el perfil común de las trabajadoras culturales, sobre cuáles son las características, estilos de vida y formatos laborales que comparten. Entre las particularidades 
que se les atribuye, aparece reiteradamente la condición precaria, realidad que parece expandirse entre las personas que integran este colectivo irregular y volátil.

Esta condición precaria puede ser entendida desde diferentes ángulos. La comisaria e investigadora Maite Garbayo-Maeztu (2018) propone su aproximación doble a partir del discurso de Judith Butler donde se marca una distinción entre precariousness y precarity (Butler 2009). La primera, apela a la posición de vulnerabilidad del sujeto entendido como cuerpo que habita y se interrelaciona con otros y el contexto. Precarity, en cambio, se refiere a las condiciones precarias de las personas que no pueden acceder a unos mínimos vitales, tanto desde lo económico como lo social. La noción de precariedad propuesta por la autora Remedios Zafra (2017) se sitúa igualmente entre estas dos acepciones y lo hace a través su protagonista, Sibila, un cuerpo de mujer atravesado por la precariedad. Zafra dibuja desde una mirada crítica feminista sujetos como cuerpos afectados, inestables, un tipo de precariedad que supera los aspectos más económico-laborales. Pérez Ibáñez y López-Aparicio (2018), por su parte, se detienen principalmente en la dimensión más económica que, según el sociólogo Guy Standing (2013), está determinada por la figura de un individuo con niveles de ingresos bajos, inestabilidad en el empleo e incertidumbre sobre su futuro laboral y personal.

En los datos que se extraen de la investigación realizada, un 46,9\% de las personas encuestadas declaran que es igual o inferior a $8.000 €$ el total de ingresos anuales que reciben por la realización de su actividad profesional, tanto las artísticas como las que no lo son (Pérez Ibáñez y López-Aparicio 2018). ¿Dónde se sitúa el umbral del sujeto precario? Con estas cifras cuesta imaginar ninguna trabajadora cultural que pueda hacerse cargo de los gastos derivados de vivienda, transporte o comida, por citar algunos. Del mismo modo parece lícito pensar en la vulnerabilidad e inestabilidad que experimenta ese cuerpo.

¿Cómo podemos medir la precariedad? ¿Dónde limitamos la tipología de trabajadora cultural que provee los datos para el estudio? ¿Dónde y desde dónde establecemos los márgenes y umbrales de la condición precaria?

$Y$ en esta incertidumbre, donde el objeto de estudio es complejo de limitar y donde toda reflexión y anclaje no es más que un discurso o visión parcial, exige de un acercamiento epistemológico que se aleje de la aparente objetividad promovida por la academia. Se trata de trabajar y generar un espacio de 
conocimiento desde las incertezas y desde un lugar que inevitablemente atraviesa la propia realidad de la autora de este artículo: trabajadora cultural que no pertenece, ni representa, ninguna asociación de artistas y/o profesionales de la cultura. Tal y como sugiere Donna Haraway (1995), no podemos escapar de aquello que somos, de nuestras situaciones históricas y sociales particulares (C. Piazzini. 2014). Y es justamente desde ese lugar, desde el conocimiento situado, desde donde se pretende abordar la discusión central de este artículo, precisamente desde una situación de vulnerabilidad que afecta directamente en la escritura y teoría que aquí se presenta.

\section{COMUNIDAD DE TRABAJADORAS CULTURALES CONSTITUÍDA FORMALMENTE}

En mayo de 2019 tuvo lugar el Seminario Internacional Prekariart cuyas ponencias se estructuraron alrededor de la pregunta ¿Es el arte un trabajo?. Organizado desde la Facultad de Bellas Artes de la universidad del País Vasco UPVI EHU, contó con expertas y artistas que trabajan e investigan contextos y conceptos de la condición precaria dentro del ámbito de las artes y la cultura. Una de las ponencias invitadas fue la que realizaron Vanesa Castro e Iñaki López, miembros fundadores de la plataforma InsultARTe. Otro de los invitados fue el artista noruego Ruben Steinum que venía en calidad de representante de dos organizaciones de artistas de Noruega: la Norwegian Visual Artists (NBK) y Young Artists Society (UKS). Si bien la pregunta sobre la necesidad de crear estructuras que aúnen intereses comunes de las trabajadoras culturales surgía de manera reiterativa durante el seminario, resulta especialmente revelador en las dos presentaciones citadas anteriormente.

La Norwegian Visual Artists (NBK) ${ }^{1}$, comparte ciertas analogías con la Unión de Artistas Contemporáneos de España². Ambos organismos recogen y se componen de otras asociaciones regionales y/o disciplinares que operan de manera independiente. Cabe señalar que en lo que se refiere al heterogéneo colectivo de artistas visuales no existe ninguna asociación que actualmente represente al contexto del País Vasco. Tanto la unión noruega como la española tienen la misión de unificar esfuerzos con el objetivo de lograr mayores y mejores condiciones para las profesionales de la cultural, y en especial para las artistas. A pesar de desarrollar su actividad en contextos muy diferentes, la necesidad de activar este tipo de proyectos colectivos y/o asociativos surge 
de un mismo lugar y premisa: desarrollo y mejoras de las condiciones laborales o regulación de mercado y políticas culturales, entre otros. Dicho de otra manera, ambos proyectos apuestan por partir de estructuras organizadas y constituidas, bien como asociaciones, sindicatos, cooperativas o cualquier otro tipo de organización registrada con el fin de reivindicar derechos y compartir preocupaciones.

Sin embargo, según datos del estudio de Pérez lbáñez y López-Aparicio (2018), de las 1.105 personas que participaron, solo un 39\% declara tener algún tipo de vínculo con algunas de las asociaciones profesionales. Las razones de esta baja afiliación es probable que sean múltiples y con certeza merecen un espacio mayor de reflexión. Pero resulta verosímil pensar que parte del argumentario tenga ver con el desencanto, la desconfianza y las fricciones internas que existen y se arrastran entre el heterogéneo grupo formado por el agente trabajador cultural. Los procesos de colectivización formal, sea cual sea el ámbito, siempre han estado sujetos a tensiones y conflictos, fruto de la propia genealogía de los procesos colectivos.

Pero como se ha citado en párrafos anteriores, la problemática sobre la creación de una institución organizada que represente los intereses de las trabajadoras culturales es una constante entre las mismas profesionales, tanto en corrillos informales como dentro del contexto más institucional y académico (congresos, seminarios, encuentros etc.).

Mientras tanto y desde un espacio de acción muy diferente, se vislumbran otro tipo de agentes que surgen desde proyectos personales llevados a lo colectivo, a menudo virtuales y que consiguen también tejer red y crear contexto. Desde una posición más alternativa, complementaria, se originan otro tipo de comunidades al margen del asociacionismo más tradicional, herencia de los movimientos obreros. InsultARTe responde precisamente a este tipo de colectividad o comunidad más informal, inestable que opera online y que, a pesar de su carácter menos estructurado, consigue ofrecer a los agentes culturales y artísticos un marco de encuentro e intercambio de conocimiento. 


\section{INSULTARTE: ALTERNATIVAS PARA EL ENCUENTRO}

InsultARTe es una comunidad abierta que utiliza una plataforma online en formato de blog como medio para representarse, hacerse visible y en cierta manera, materializarse. En su plataforma web -www.insultarte.net- se autodefine como una comunidad que denuncia convocatorias, bases de festivales, y otras propuestas que se aprovechan de los/as trabajadores/as culturales; es decir, un lugar para visibilizar todas las prácticas abusivas e insultantes que suceden en el ámbito de la cultura. En las páginas aparecen mensajes y contenidos relativos a las situaciones denunciadas, pero no se reduce únicamente a la denuncia, sino que también ofrece un amplio abanico de posibilidades, herramientas y consejos dirigidos tanto a artistas como instituciones con el fin de promover las buenas prácticas en el ámbito de la producción cultural y/o artística.

El blog se compone de más de 200 entradas que se llevan acumulando como un archivo organizado cronológicamente desde 2008. Este proyecto fue creado y sigue siendo gestionado por las artistas Vanesa Castro e Iñaki López que en paralelo operan igualmente como colectivo bajo el nombre Fur Alle Falle $^{3}$. Esta doble identidad refleja la necesidad o deseo de separar ambos proyectos, ambas actividades que, aunque coincidentes desde el punto de vista y posicionamiento político frente al contexto cultural, operan desde dos lugares distintos: la mediación/consultoría en el caso de InsultARTe; y desde la producción artística en el de Fur Alle Falle. En lo referente a InsultARTe, Castro y López no pretenden esconderse, sino más bien permanecer en la sombra para darle protagonismo a las usuarias que intervienen y conforman toda esta comunidad heterogénea e intangible.

La forma de intervenir y participar en el proyecto InsultARTe se realiza, por un lado, a través del apartado de comentarios habituales en las estructuras blog y, por otro, por medio del mail que reciben Castro y López, normalmente con el testimonio y denuncia de alguna situación que los sujetos han experimentado. InsultARTe también encuentra en las redes sociales otro lugar para el diálogo e interacción entre usuarias. Ha formado un grupo en Facebook que ya cuenta con 1412 usuarias y una cuenta Twitter con 3121 seguidoras. No existen registros y, por tanto, son datos que pueden variar, que no recogen ni nombres, ni perfiles, ni tampoco se pretende. 
La naturaleza de este tipo de comunidades más inestables, líquidas, no tiene por qué responder a una falta de recursos para crear otro tipo de comunidad más formal. La génesis de InsultARTe probablemente no surge de una intención tan planificada, si que más bien se ha ido creando en la medida que han ido aumentando las intervenciones de los usuarios y con el mantenimiento y dinamización de las dos personas fundadoras. De tal forma que, tras diez años en línea, sigue siendo una comunidad variable y parcial, pero que a pesar de su tamaño también presenta ciertos puntos fuertes como son la capacidad de inmediatez, la actualización compartida y un lenguaje más cotidiano y a menudo irónico que genera cierta cercanía con toda persona que se acerca a su lectura.

InsultARte no pretende recoger y realizar un estudio objetivo en torno a las buenas y malas prácticas basándose en rigurosas metodologías de análisis. No obstante, tras 10 años de actividad, es la propia comunidad quien, en cierta manera, ha legitimado la información que aparece. Legitimación que supera el anonimato de la red y que se establece igualmente a través de encuentros, ponencias, talleres y charlas a los que centros de arte y cultura y universidades a los que InsultARte ha sido reiteradamente invitado. Además de la citada Universidad del País Vasco, la lista de instituciones se amplía a la Universidad Complutense de Madrid, el CGAC (Centro Galego de Arte Contemporánea) o Matadero Centro de Creación Contemporánea.

\section{ESTUDIO REALIZADO POR INSULTARTE}

En los resultados del estudio anteriormente mencionado, quedan expuestas las dificultades que las investigadoras Pérez Ibáñez y López-Aparicio hallaron desde la misma fase inicial del planteamiento respecto al censo de las trabajadoras culturales. Determinar el sujeto de estudio, aquel que responde a la categoría de trabajadora cultural, es ciertamente imprescindible para realizar un estudio riguroso sobre precariedad en el contexto cultural. La investigación llevada a cabo por los dos autores se enmarca en el campo académico, y por tanto, requiere de cierta metodología, un protocolo que a duras penas puede dejarse de lado. Existen igualmente otros estudios desarrollados tanto desde el ámbito académico como desde los estudios sociológicos. De la mano de la Asociación de Artistas Visuales de Cataluña, La dimensión económica de las artes visuales en España (2006); el estudio realizado por Asociación de Artista 
visuales de Valencia, Castellón y Alicante AVAAC (2013); o el de la empresa ARTimetría que siguiendo un encargo del Gobierno Vasco recoge datos y formula propuestas con el objetivo de realizar un diagnóstico fiel a la realidad cultura del territorio (2009). Todos estos estudios sirven y ayudan a ilustrar las condiciones de precariedad del ámbito cultural.

Desde otro lugar, menos cuantitativo, InsultARTe también ha realizado su propio estudio a partir de los sujetos usuarios de la web y redes sociales de su comunidad virtual a finales del 20144. Con la intención de desentramar y cuantificar la diversidad de situaciones de precariedad que viven las trabajadoras de la cultura, distribuyeron una encuesta con 19 preguntas a través de sus canales. Llegaron a contestar 249 personas o profesionales de la cultura. La segunda pregunta del cuestionario hacía referencia a las categorías profesionales que se engloban dentro del trabajo cultural por medio de la siguiente y aparente sencilla pregunta: ¿Qué palabra eliges? Las respuestas posibles eran múltiples. Toda una amalgama de posibilidades profesionales de las cuales las más votadas fueron la de artista, gestor/a, creador/a, investigador/a y comisario/a, en este orden, y las menos, por su parte, fueron las de autora, ninguna, productor/a, agente o artesano/a. InsultARTe toma una posición clara cuando construye de este modo la pregunta. No desea perder el tiempo en definiciones, ni limitaciones de funciones.

De esta pregunta se extraen todas las posibilidades profesionales que existen en los trabajos culturales y cómo cada sujeto decide en qué lugar o a través de qué nomenclatura se siente más cómoda. ¿Cuántas veces una no sabe qué contestar frente a la habitual pregunta sobre a qué te dedicas o qué eres? La enunciación que InsutARTe decide formular libera de ese bloqueo al intentar proyectar cierta ligereza en una cuestión que no siempre lo es.

La segunda cuestión que puede extraerse de esta pregunta y sus consiguientes respuestas es la de la necesidad de reconocer los dobles oficios, la multitarea, los dobles o triples trabajos que una trabajadora cultural tiende a realizar. La precariedad económica, los bajos niveles de remuneración o la inestabilidad de los trabajos culturales a menudo sitúan a un mismo sujeto llevando a cabo tareas de comunicación, producción, mediación, creación y/o gestión. De hecho, se podría realizar otro interesante estudio a partir de la hipótesis de que muchas de las artistas que combinan su práctica con tareas de gestión y producción acaban abandonando la creación debido a que las tareas de intermediación como la gestión, comunicación o producción cultural, están en general mejor remuneradas y cuentan con mejores condiciones labora- 
les y emocionales. "Ten en cuenta que en un evento en el que todos cobran (los que organizan, los que trabajan limpiando, los técnicos, etc.) tú también debes cobrar." (InsultARTe 2011). Con esta afirmación tan directa explican en InsultARTe una de las paradojas habituales del ámbito cultural, donde todas las profesionales que rodean el hecho artístico (gestoras, productoras, limpiadoras, equipo técnico etc.) cobran y lo hacen en mejores condiciones que la artista que provee los contenidos. Por si fuera poco, a esta situación de multitarea en lo cultural, hay que añadirle frecuentemente otro trabajo que nada o poco tiene que ver con la cultura.

La condición multitarea afecta directamente a la disposición del tiempo. Hecho que posibilita pensar en sujetos sin tiempo suficiente para poder invertirlo entre otras cosas, en organizarse, en crear estructuras más formales, estables y que puedan operar como un agente legitimado para, por ejemplo, establecer un diálogo productivo con otras estancias más políticas y sociales que favorezcan el cambio.

"También, y a modo de auto-crítica, quizás los artistas deberíamos responsabilizarnos y no aceptar trabajar con las instituciones que nos traten como si nos estuviesen haciendo un favor, como si tuviésemos que mostrarnos agradecidos por las limosnas que nos dan".

(Nuria Güell 2019)

Asimismo, y tal y como expone la artista Nuria Güell, InsultARTe también apunta hacia otras posibles causas que repercuten en la falta de algún tipo de estructura colectiva y organizada que defienda los intereses del gremio, si es que es posible denominarlo así. Se trata de la autoexplotación consciente, o no, que las trabajadoras de la cultura ejercen sobre sí mismas. Bajo la falsa idea de sentirnos afortunadas por poder hacer lo que nos gusta (proyecto artístico culturales), alimentar un ego y un reconocimiento público y, probablemente también, por tener ciertos prejuicios en reconocernos vulnerables y precarias, se suelen aceptar las condiciones de precariedad que se despliegan en muchas de las becas, premios, ayudas u otro tipo de convocatorias artístico-culturales (Zafra 2017). InsultARTe dedica gran parte de su esfuerzo divulgativo en generar una toma de conciencia entre las propias trabajadoras, una visión crítica hacia muchas de las convocatorias que surgen tanto desde las instituciones públicas como privadas. Su web y redes sociales están plagadas de esas prácticas abusivas que no hacen más que seguir consolidando un estado de precariado constante entre las trabajadoras culturales. 


\section{CONCLUSIONES}

¿Necesitamos medir y contabilizar la situación laboral, económica, psicológica o social de la trabajadora cultural para poder enfrentarnos a ella? Resulta pertinente conocer y diagnosticar las posibles realidades, no obstante, sería conveniente que no fuera una condición sine qua non para poder abordar la cuestión de la precariedad en el arte y la cultura. Plataformas como la de InsultARTe se adelantan a los datos precisos, no esperan tener una definición de la categoría de lo que incluye o excluye la noción de trabajadora cultural. Desde los márgenes ofrece la posibilidad de trazar una hipotética comunidad virtual, que aúne un porcentaje de afiliadas superior al que apuntaban Pérez lbáñez y López-Aparicio de un 39\% del total.

InsultARTe surge desde una convicción y una necesidad particular de las artistas Iñaki López y Vanesa Castro con la pretensión de generar, en otros, una mirada crítica entre las mismas trabajadoras culturales. Se trata de una iniciativa más informal, procesual, abierta, con un lenguajes cotidiano, mutable y virtual que no necesita más que de usuarios anónimos que compartan sus propias experiencias y conocimientos. Representa una alternativa que comulga con las dinámicas de la red, de internet, sin en ningún caso pretender sustituir otro tipo de estructura más formal. InsultARTe no aspira más que a formar una comunidad informe, virtual, sin las posibles cargas burocráticas que conlleva el registro y funcionamiento de una asociación, sindicato o cualquier otro modelo organizacional registrado. Desde ese lugar más arbitrario, líquido, es posible hacer uso de un lenguaje menos estricto, valerse del humor y lograr permear desde una perspectiva más divulgativa para lograr su objetivo, que como se ha mencionado, no es más que potenciar el espíritu crítico de las trabajadoras culturales.

Pese a la utilidad e interés de comunidades como la que representa InsultARTe, no pueden ser consideradas como un interlocutor lo suficientemente legitimado ni operativo. InsultARTe se representa a sí mismo y es parte de su idiosincrasia la que le da la libertad de no tener que hablar desde las certezas, como voz colectiva, ni en nombre de todas las trabajadoras culturales. Trabaja desde los márgenes, desde la condición precaria, sin recursos para actualizar, gestionar los contenidos y comprobar cada información que recibe. Y a pesar todo, resulta un proyecto que dentro de su espacio se ha ganado la confianza de muchas usuarias y ha conseguido ser legitimada por las instituciones más formales (academia, institución cultural pública). 


\section{Referencias bibliográficas}

AAVC (Associacio d'Artistes Visuals de Catalunya). 2006. La dimensión económica de las artes visuales en España. Barcelona: Associació d'Artistes Visuals de Catalunya

ARTImetría. 2009. Valoración económica del trabajo del creador-a en las artes visuales: Informe final. Dir., Antoni Laporte; coord., Joaquina Bobes. Vitoria-Gasteiz: Gobierno del País Vasco. https://avvac.files.word.com/2014/01/artimetria-pv.pdf

AVVAC (Artistes Visuals de València, Alacant i Castelló). 2013. "Informe sobre la situación de los artistas visuales". Avvac.wordpress.com 21 marzo. https://avvac.word.com/2013/03/21/ estatuto-del-artista-2012/

Butler, Judith. 2009. Marcos de guerra: Las vidas lloradas. Traducción de Benardo Moreno Carrillo. Barcelona: Paidós

Garbayo Maeztu, Maite. 2018. "Maternidad, arte y precariedad: estrategias desde la vulnerabilidad". Arte y Políticas de Identidad 19: 68-82

Güell, Núria. 2019. “¿Cómo regular la práctica artística a través de un estatuto profesional que responde a las necesidades de todos los artistas?". Ponencia de "Prekariart", Seminario Universidad del País Vasco (UPV/EHU), Bilbao, 8-10 mayo

Haraway, Donna J. 1995. Ciencia, cyborgs y mujeres: La reinvención de la naturaleza. Traducción de Manuel Talens. Madrid: Cátedra

InsultARTe. 2011. "Consejos para creadores, artistas, productores culturales, etc". InsultARTe (blog) 13 abril. http://www.insultarte.net/p/ideas.html?spref=bl

Pérez Ibáñez, Marta \& Isidro López Aparicio. 2018. "Actividad artística y precariedad: la situación económica de los/las artistas en España a partir de un estudio global”. En VIII Workshop en Economía y Gestión de la Cultura, Universidad de Sevilla

Piazzini Suárez, Carlo Emilio. 2014. "Conocimientos situados y pensamientos fronterizos: Una relectura desde la universidad". Geopolíticas 5(1): 11-33

Standing, Guy. 2013. El precariado: Una nueva clase social. Traducción de Juan Mari Madariaga. Barcelona: Pasado \& Presente

Zafra Alcaraz, Remedios. 2017. El entusiasmo: Precariedad y trabajo creativo en la era digital. Barcelona: Anagrama

\section{Notas}

${ }^{1}$ Association of Norwegian Visual Artists. https://www.norskebilledkunstnere.no/english/

2 Unión de Artistas Contemporáneos de España. https://unionac.es/

${ }^{3}$ Für Alle Fälle - Para todas las ocasiones (Vanesa Castro \& Iñaki López). http://www.furallefaIle.com/

${ }^{4}$ Encuesta realizada en 2014 por InsutARTe: https://insultarte.typeform.com/report/hoGgRX/ $\mathrm{H} 8 \mathrm{Yw}$ 